\title{
Contexto de trabalho e Síndrome de Burnout na equi- pe de enfermagem da Estratégia Saúde da Família
}

\author{
Contexto laboral y el síndrome de burnout en el equipo de \\ enfermería de Salud Estratégica de la Familia \\ Work Context and Burnout syndrome in the nursing team \\ of the Family Health Strategy
}

Renata Bernardes Lacerda ${ }^{1}$, Maria Beatriz Guimarães Ferreira ${ }^{2}$, Carolina Feliciana Bracarense ${ }^{3}$, Lidiane Vieira de Sene ${ }^{4}$, Ana Lúcia de Assis Simões. ${ }^{5}$

${ }^{1}$ Acadêmica do Curso de Enfermagem da Universidade Federal do Triângulo Mineiro. Uberaba, MG, Brasil ${ }^{2}$ Enfermeira. Mestre. Doutoranda pelo Programa de Pós-Graduação em Enfermagem Fundamental da Escola de Enfermagem de Ribeirão Preto da Universidade de São Paulo. Ribeirão Preto-SP. Brasil.

${ }^{3}$ Enfermeira. Mestre. Professora Substituta do Curso de Graduação em Enfermagem da Universidade Federal do Triângulo Mineiro. Uberaba, MG, Brasil.

${ }^{4}$ Enfermeira. Mestre. Membro do Grupo de Estudo e Pesquisa em Gerenciamento em Enfermagem e Saúde da Universidade Federal do Triângulo Mineiro. Uberaba, MG, Brasil.

${ }^{5}$ Enfermeira. Doutora em Enfermagem. Professora Associada do Curso de Graduação em Enfermagem e do Programa de Pósgraduação em Atenção à Saúde da Universidade Federal do Triângulo Mineiro. Uberaba, MG, Brasil.

Cómo citar este artículo en edición digital: Lacerda, R.B., Ferreira, M.B.G., Bracarense, C.F., Sene, L.V. y. Simões, A.L.A.

(2016). Contexto de trabalho e Síndrome de Burnout na equipe de enfermagem da Estratégia Saúde da Família

Cultura de los Cuidados (Edición digital), 20, 44. Disponible en:< http://dx.doi.org/10.14198/cuid.2016.44.08>

Correspondencia: Rua João Pinheiro No2800 Bairro: Boa Vista Cidade: Uberaba/MG

Correo electrónico: renatablacerda@hotmail.com

Recibido: 06/07//2015; Aceptado: 05/10/2015

Este trabalho recebeu fomento da Fundação de Amparo à Pesquisa do Estado de Minas Gerais (FAPEMIG), com o número do processo APQ01417-12.

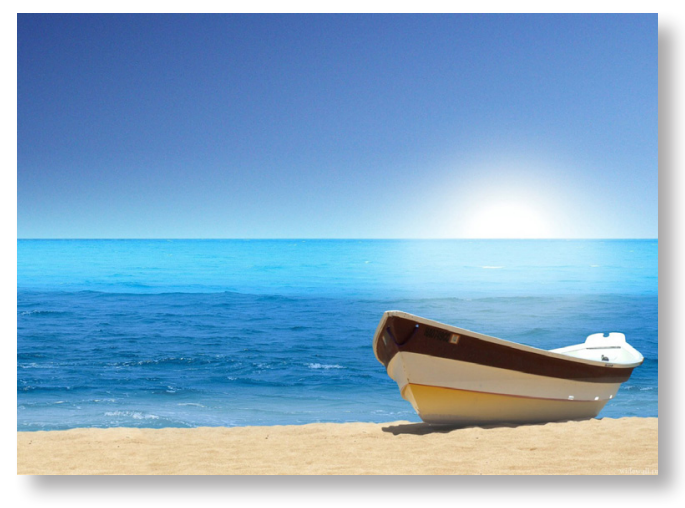

ABSTRACT

Purpose: Investigate the relationship between the working conditions and the Burnout Syndrome of nursing professionals of Family Health Strategy town of Minas Gerais.

Methods: Cross-sectional study with a quantitative approach. The study group was composed of 50 nursing professionals who answered the questionnaire of sociodemographic and professional, Work Context Assessment Scale (EACT) and Maslach Inventory Burnout. Exploratory and bivariate analyzes were used at 5\% significance level.

Results: The dimension Emotional Exhaustion was significantly correlated with all factors of EACT; Depersonalization presented diference with the Socio-professional Relations and Work Organization; Decreased Personal accomplishment correlated with Labour Organization.

Conclusion: Worst are the conditions and organization of work and the socio-professional relations, the greater the possibility of dissatisfaction, development of nonchalance attitudes, disease and emotional exhaustion 
among nursing professionals in primary care.

Keywords: Burnout, Professional; Working Conditions; Family Health Strategy; Nursing, Team.

\section{RESUMEN}

Objetivo: Investigar la relación entre el medio ambiente de trabajo y el personal de enfermería de la familia de un pueblo minero Estrategia de Salud Síndrome de Burnout.

Método: Estudio transversal con un enfoque cuantitativo. El grupo de estudio estuvo compuesto por 50 profesionales de enfermería que respondieron al cuestionario sobre la escala sociodemográfica y profesional Evaluación Contexto Trabajo (EACT) e Inventario Síndrome de Burnout. Se utilizaron análisis exploratorios y bivariadas, considerando un nivel de significación del 5\%.

Resultados: escala de Agotamiento emocional correlacionó significativamente con todos los factores de EACT; La despersonalización era diferente de las relaciones socioprofesional y la organización del trabajo; Disminución de Cumplimiento correlacionado con la Organización del Trabajo.

Conclusión: Lo peor son las condiciones y la organización del trabajo y las relaciones socioeconómicas, mayor será la posibilidad de insatisfacción, el desarrollo de actitudes de endurecimiento, la enfermedad y la atención primaria emocional de enfermera agotamiento.

Palabras clave: Burnout Profesional; Condiciones de trabajo; Estrategia Salud de la Familia; El personal de enfermería.

\section{RESUMO}

Objetivo: Verificar a relação entre o contexto de trabalho e a Síndrome de Burnout dos profissionais de enfermagem da Estratégia Saúde da Família de um município mineiro.
Método: Estudo seccional, de abordagem quantitativa. $\mathrm{O}$ grupo de estudo foi composto por 50 profissionais de enfermagem que responderam o questionário de caracterização sociodemográfica e profissional, Escala de Avaliação de Contexto de Trabalho (EACT) e Inventário da Síndrome de Burnout. Foram utilizadas análises exploratória e bivariada, considerando nível de significância de 5\%.

Resultados: A dimensão Exaustão Emocional apresentou correlações significativas com todos os fatores da EACT; Despersonalização apresentou diferença com as Relações Socioprofissionais e Organização do Trabalho; Diminuição da Realização Pessoal correlacionou-se com Organização do Trabalho.

Conclusão: Quanto pior sejam as condições e organização do trabalho e as relações socioprofissionais, maior a possibilidade de insatisfação, desenvolvimento de atitudes de insensibilidade, adoecimento e exaustão emocional do profissional de enfermagem da atenção primária.

Palavras chave: Esgotamento Profissional; Condições de Trabalho; Estratégia Saúde da Família; Equipe de Enfermagem

\section{INTRODUÇÃO}

O Programa Saúde da Família (PSF) surgiu no Brasil, como uma estratégia de reorientação do modelo assistencial a partir da atenção básica. Este programa desenvolve estratégias de acolhimento do indivíduo, da família e comunidade, fora do ambiente hospitalar (Santos et al., 2011).

Mesmo que classificado como um Programa, o PSF não incorpora as características dos demais programas idealizados pelo Ministério da Saúde. O PSF possui um caráter inovador, caracteriza-se como uma estratégia, uma vez 
que promove a organização das ações em um território definido e tem a intenção de enfrentar e solucionar os problemas identificados. Diante disso, em 1998, o Programa Saúde da Família passou a ser compreendido e denominado como Estratégia Saúde da Família (ESF) (Silva-Roosli; Athayde, 2011).

A ESF é composta por uma equipe multiprofissional: agentes comunitários de saúde, auxiliares de consultório dentário, dentistas, enfermeiros, médicos e técnicos de enfermagem (Brasil, 2011). Estes profissionais desenvolvem ações sistematizadas e contínuas, com práticas educativas e preventivas voltadas ao acolhimento humanizado do indivíduo, família e coletividade (Fernandes; Backes, 2010).

Dentre estas atividades realizadas pela equipe de saúde da família compete ao enfermeiro: realizar consultas de enfermagem, solicitar exames complementares, prescrever medicações; de acordo com os aspectos éticos, legais e protocolos existentes, planejar, gerenciar, coordenar e avaliar as ações desenvolvidas pelos agentes comunitários de saúde e equipe de enfermagem, bem como, contribuir e participar das atividades de Educação Permanente (Brasil, 2011).

Já os técnicos em enfermagem são responsáveis por: participar das atividades de assistência básica realizando procedimentos regulamentados no exercício de sua profissão na ESF e, quando indicado ou necessário, no domicílio; realizar ações de educação em saúde a grupos específicos e a famílias em situação de risco, conforme planejamento da equipe (Brasil, 2011).

Os profissionais de saúde da ESF desenvolvem suas atividades junto à comunidade, onde a realidade do indivíduo fica muito próxima, os problemas são de diversas ordens e as limitações internas/externas inúmeras. Essas par- ticularidades expõem os trabalhadores a riscos físicos e/ou psicossociais, os quais podem desencadear um processo de estresse ocupacional (Santos; David, 2011).

No que tange aos profissionais de enfermagem, os principais riscos psicossociais são: falta de preparo e capacitação, sobrecarga de papéis, longas horas no trabalho, conflito no trabalho em equipe, dificuldade para conciliar trabalho e família, recursos materiais e humanos insuficientes, além das condições de trabalho precárias (Santos; David, 2011).

As condições de trabalho atuam direta ou indiretamente na qualidade de vida dos profissionais e nos resultados obtidos. $\mathrm{O}$ excesso de carga horária e/ou atividades, podem ocasionar estresse para o profissional devido as múltiplas e exaustivas funções. Essa sobrecarga pode ser causadora de esgotamento físico e/ou mental. O profissional inserido em ambiente de trabalho inadequado tende a desenvolver sinais de estresse tendo prejuízo no desempenho profissional, com comprometimento da qualidade da assistência prestada (Holmes et al., 2014).

$\mathrm{O}$ estresse é a resposta às demandas do ambiente externo. $\mathrm{O}$ modo como a pessoa lida com as circunstâncias geradoras de estresse pode levá-la ao esgotamento profissional, indicativo da Síndrome de Burnout, que pode desencadear reações como esgotamento psíquico (Santos; David, 2011).

Síndrome de Burnout é a resposta a um estresse crônico, típico de trabalho diário, que afeta diretamente o desempenho de tarefas, relacionamento interpessoal, produtividade e, até mesmo, a qualidade de vida no trabalho, do indivíduo e da organização, devido à exposição direta com comunidade, e à extensa jornada de trabalho em condições, muitas vezes, desfavoráveis (França et al., 2012). 
São características desta Síndrome: Exaustão Emocional, falta de entusiasmo em razão do esgotamento; a Despersonalização, que ocorre quando o profissional passa a tratar de forma fria e distante as pessoas; e, a Baixa Realização no Trabalho, tendência em se avaliar de forma negativa (França; Ferrari, 2012). Dentre as consequências advindas do Burnout à saúde destacam-se os altos índices de absenteísmo e isolamento, ocasionados por alterações emocionais, alterações físicas e problemas de saúde (Trindade et al., 2010).

Diante do exposto emergiu o seguinte questionamento: Quais os fatores do contexto de trabalho influenciam no aparecimento da Síndrome de Burnout? Os objetivos da presente investigação são: caracterizar os profissionais de enfermagem da ESF do município de Uberaba, Minas Gerais (MG), Brasil, segundo as variáveis sócio demográficas e profissionais; mensurar o contexto de trabalho e a Síndrome de Burnout, bem como verificar a relação entre o contexto de trabalho e a Sindrome de Burnout desses profissionais.

\section{MÉTODO}

Trata-se de uma pesquisa observacional, seccional, com abordagem quantitativa, realizada no município de Uberaba - MG. Participaram do estudo os profissionais de enfermagem vinculados à ESF, técnicos em enfermagem e enfermeiros. No momento em que ocorreu a coleta de dados, o município estruturava a atenção primária em 50 Equipes de Saúde da Família, das quais 46 estavam na zona urbana e quatro na zona rural.

Trata-se de um estudo que pertence a um projeto maior e, portanto, foram selecionados todos os profissionais de enfermagem, resultando em um tamanho amostral $(n=50)$, entre enfermeiros e técnicos em enfermagem.
A coleta foi realizada no período de setembro de 2013 a janeiro de 2014, por meio de encontros previamente agendados com os profissionais e a auto aplicação de um questionário semiestruturado de caracterização sociodemográfica e profissional, elaborado pelos autores e de dois instrumentos já validados, sendo: Inventário da Síndrome de Burnout traduzido e validado por Tamayo (1997) e Escala de Contexto de Trabalho (EACT), proposto por Ferreira e Mendes (2008).

O Inventário da Síndrome de Burnout tem a finalidade de estimar o sentimento do profissional pelo trabalho, e tem como base três dimensões: Exaustão Emocional, que versa sobre fadiga do profissional, o esgotamento físico e mental, bem como a sensação do mesmo ter chegado no limite de suas possibilidades, avaliada por dez itens; Diminuição da Realização Pessoal, alusivo ao bem-estar do indivíduo com o trabalho, avaliado por sete itens; e Despersonalização, a qual investiga o desenvolvimento de atitudes de insensibilidade, frieza e impessoalidade ao sofrimento dos usuários, avaliado por dois itens.

A EACT busca compreender as representações que os profissionais têm sobre o contexto de trabalho no qual estão inseridos, para isso tal escala é alicerçada em três fatores: Condições de trabalho, a qual representa a infraestrutura do ambiente de trabalho, a disponibilidade de recursos materiais para executar a atividade laboral, avaliada por dez itens; Organização do Trabalho, que reflete as normas e rotinas da instituição, a divisão e o ritmo de trabalho, avaliada por nove itens, e Relações Socioprofissonais, que refere-se à comunicação profissional e os modelos de gestão, avaliado por 11 itens.

Ambas escalas são do tipo Likert de cinco pontos, variando entre os intervalos 1 , que 
equivale a nunca, a 5, que equivale a sempre. $\mathrm{O}$ escore nos fatores foram estimados pela média dos pontos atribuídos pelos participantes a cada item que compõe as dimensões.

Os participantes assinaram o Termo de Consentimento Livre e Esclarecido, no momento que consentiram em participar do estudo. A coleta foi realizada em momentos, os quais foram programados antecipadamente com os gestores das Unidades de Saúde, por uma equipe de investigadores, entre setembro de 2013 e janeiro de 2014.

Para proceder a análise dos dados foi realizado dupla digitação, com posterior validação e utilizado o aplicativo Statistical Package for the Social Science (SPSS), versão 21. Primeiramente, os dados foram submetidos à análise exploratória por meio de frequências simples, medidas de posição (mediana e média) e de variabilidade (amplitude e desvio padrão). Na análise bivariada, utilizaram-se os testes $\mathrm{t}$ de Student para as variáveis com dois grupos, e correlação de Pearson para quantificar as relações entre as variáveis quantitativas.

A pesquisa obteve autorização da Secretaria Municipal de Saúde de Uberaba-MG, bem como a aprovação do Comitê de Ética em Pesquisa da Universidade Federal do Triângulo Mineiro, mediante parecer no 2244.

\section{RESULTADOS}

Do total de 50 (100\%) profissionais de enfermagem estudados, 26 (52,0\%) eram técnicos em enfermagem e 24 (48,0\%) enfermeiros. Destes, 47 (94,0\%) eram do sexo feminino e três $(6,0 \%)$ do sexo masculino. Apresentaram idade média de 37,87 anos, com desvio padrão de 8,64, idade mínima de 27,21 anos e máxima de 59,13 anos. No que concerne a situação conjugal, 26 (52,0\%) eram casados ou em união estável e $24(48,0 \%)$ não possuíam com- panheiro. Para realizar a avaliação econômica foi utilizado o Critério de Classificação Socioeconômica do Brasil da Associação Brasileira de Empresas de Pesquisa, evidenciando a totalidade (100\%) dos profissionais pertencentes a classe $\mathrm{C}$ ou mais.

Em relação ao nível escolaridade, 23 $(46,0 \%)$ tinham ensino médio completo e 27 $(54,0 \%)$ possuíam curso superior. Quanto à formação complementar, 24 (48,0\%) realizaram curso de especialização, um $(2,0 \%)$ havia concluído o curso de mestrado e 25 (50,0\%) não possuíam formação complementar. Dentre os participantes da pesquisa, $26(52,0 \%)$ estudaram em instituição de ensino pública e 24 (48,0\%) em instituição privada.

No que se refere ao número de vínculos empregatícios, 40 (80,0\%) entrevistados possuíam vínculo único e 10 (20,0\%) duplo vínculo. O tempo médio de profissão na ESF foi de 118,48 meses, com desvio padrão de 66,66, tempo mínimo de 5 meses e máximo de 336 meses. No que tange ao tempo de trabalho com o mesmo gerente, a média foi de 17,82 meses, com desvio padrão de 25,77, tempo mínimo de 3 meses e máximo de 156 meses.

Observa-se, pela tabela I, que no Inventário de Burnout, a dimensão Diminuição da Realização Pessoal apresentou o maior escore médio e Exaustão Emocional, o menor escore médio. Quanto aos fatores da EACT, a que apresentou maior escore médio foi a Organização do Trabalho, enquanto Relações Socioprofissionais apresentou o menor escore médio. No que se refere a avaliação da consistência interna dos instrumentos, expressa pelo coeficiente Alfa ( $\alpha$ ) de Cronbach, evidenciou-se boa consistência interna, com valores variando entre 0,68 e 0,91 .

Tabela I - Valores mínimos e máximos, escore médio, mediana, desvio padrão e coefi- 
ciente $a$ de Cronbach das dimensões da "Escala de Avaliação do Contexto de Trabalho" e das dimensões da síndrome de Burnout dos profissionais de enfermagem da ESF de um município do interior de Minas Gerais, $2014(\mathrm{n}=50)$.
$\mathrm{Na}$ escala do Contexto de trabalho os itens que apresentaram menores médias foram o 24 $(\chi=2,06)$ (Existem dificuldades na comunicação chefia- subordinado?), $30 \quad(\chi=2,14)$ (Falta apoio das chefias para o meu desenvolvimento profissional?) e 29 $(\chi=2,20)$ (As informações de que preciso para executar minhas tarefas são de difícil acesso?). Já os itens 3 $(\chi=3,72)$ (A cobrança por resultados é presente?), $1 \quad(\chi=3,66)$ (O ritmo de trabalho é acelerado?) e 2 $(\chi=3,58) \quad$ (As tarefas são cumpridas com pressão temporal?)

Ao analisar os itens do Inventário de Burnout nota-se que os itens $19(\chi=4,16)$ (Eu tenho realizado muitas coisas importantes neste trabalho), $7(\chi=4,06)$ (Eu trato de forma adequada os problemas dos meus pacientes) e 21 $(\chi=3,84)$ (No meu trabalho, eu lido com os problemas emocionais com calma) obtiveram as maiores médias. Em contrapartida, os itens $5(\chi=1,52)$ (Eu sinto que eu trato alguns dos meus pacientes como se fossem objetos), 15 $(\chi=1,62)$ (Eu não me importo realmente com o que acontece com alguns dos meus pacientes) e $20(\chi=2,10)$ (No meu trabalho, eu me sinto como se estivesse no final do meu limite) apresentaram as menores médias.

Os itens 7, 19 e 21 pertencem à dimensão Diminuição da Realização Pessoal e os itens 5 e 20 à Exaustão Emocional. A descrição dos escores médios dos itens é confirmada pelos maiores e menores escores médios das dimensões, respectivamente, Diminuição da Realização Pessoal e Exaustão Emocional. apresentaram a maior média.

Evidencia-se que os itens 1, 2 e 3 pertencem ao fator Organização do Trabalho, e os itens 24, 29 e 30 ao Relações Socioprofissionais. A descrição corrobora com os dados apresentados na tabela 1, a qual evidencia o fator Organização do Trabalho com maior escore médio, enquanto Relações Socioprofissionais, o menor escore médio.

Na tabela II a análise bivariada entre Síndrome de Burnout e contexto de trabalho, avaliado pela EACT, foi utilizada correlação de Pearson, sendo a magnitude da correlação interpretada em fraca (para $r$ entre 0,00-0,29); moderada ( $\mathrm{r}$ entre $0,30-0,49)$ e forte ( $\mathrm{r}$ entre $0,50-1,00)$. Os testes foram considerados significativos quando $\mathrm{p}<0,05$.

Tabela II - Análise da Correlação de "Pearson", tendo como desfechos as dimensões da síndrome de Burnout dos profissionais de enfermagem da ESF de um município do interior de Minas Gerais, 2014. 


\begin{tabular}{lllllll}
\hline $\begin{array}{l}\text { Contexto de } \\
\text { trabalho }\end{array}$ & $\begin{array}{l}\text { Exaustão } \\
\text { emocional }\end{array}$ & Despersonalização & \multicolumn{3}{c}{$\begin{array}{c}\text { Diminuição } \\
\text { da } \\
\text { realização } \\
\text { pessoal }\end{array}$} \\
$\begin{array}{l}\text { Síndrome } \\
\text { de Burnout }\end{array}$ & $\mathbf{R}$ & $\mathbf{P}$ & $\mathbf{r}$ & $\mathbf{P}$ & $\mathbf{r}$ & $\mathbf{P}$ \\
\hline $\begin{array}{l}\text { Condições de } \\
\text { trabalho }\end{array}$ & 0,35 & 0,01 & 0,14 & 0,33 & 0,26 & 0,07 \\
\hline $\begin{array}{l}\text { Organização do } \\
\text { trabalho }\end{array}$ & 0,47 & $<0,001$ & 0,31 & 0,03 & 0,38 & 0,007 \\
\hline $\begin{array}{l}\text { Relações } \\
\text { Socioprofissionais }\end{array}$ & 0,51 & $<0.001$ & 0,29 & 0,04 & 0,19 & 0,18 \\
\hline
\end{tabular}

isto evidencia que a profissão enfermagem, ainda, é mais frequente entre as mulheres, assemelhando-se aos resultados encontrados por Linch e Guido (2011). França e

No que concerne a esta análise bivariada, foram verificadas correlações estatisticamente significativas entre a dimensão Exaustão Emocional e todos os fatores da EACT, Condições de Trabalho ( $r=0,35 ; \mathrm{p}=0,01)$, Organização do Trabalho ( $\mathrm{r}=0,47$; $\mathrm{p}<0,001)$ e Relações Socioprofissionais $(r=0,51 ; p<0,001)$. Tais resultados demonstram que quanto pior sejam as condições de trabalho, organização do trabalho e as relações socioprofissionais, maior a possibilidade do adoecimento do profissional e sua exaustão emocional.

A dimensão Despersonalização apresentou diferença estatística e correlação fraca entre as Relações Socioprofissionais $(\mathrm{r}=0,29 ; \mathrm{p}=0,04)$ e correlação moderada com o fator Organização do Trabalho $(r=0,31 ; p=0,03)$. O que evidencia que um ambiente laboral com relações socioprofissionais e organização do trabalho não satisfatórias favorece o desenvolvimento de atitudes de insensibilidade.

A dimensão Diminuição da Realização pessoal obteve-se correlação forte com o fator Organização do Trabalho ( $\mathrm{r}=0,38 ; \mathrm{p}=0,007)$, evidenciando que quanto pior a organização do trabalho e as políticas institucionais, maior a probabilidade do indivíduo não se realizar e se sentir insatisfeito com o trabalho desempenhado.

\section{DISCUSSÃO}

Dentre a caracterização sóciodemográfica e profissional há a prevalência do sexo feminino,
Ferrari (2012) realizaram uma pesquisa com enfoque na Síndrome de Burnout nos profissionais de enfermagem da rede hospitalar, os resultados desta apontaram que a maioria dos entrevistados estavam na faixa etária de 20-40 anos e eram casados, tais achados corroboram com o presente estudo.

Os resultados mostraram que a maioria dos profissionais de enfermagem não possuía outro emprego na área, desfecho este infrequente, tendo em vista que é comum prática do duplo vínculo empregatício na enfermagem, devido aos baixos salários, condição esta que ocasiona ao profissional estresse e baixa qualidade de vida (França et al.,2012)

Os sujeitos pesquisados apresentaram tempo médio de profissão de aproximadamente 10 anos, há controvérsias quanto o surgimento da síndrome estar relacionada ao tempo de trabalho ou a pouca experiência do trabalhador devido ao risco da instabilidade e a necessidade da sua inserção no grupo (Trindade; Lautert, 2010). Entretanto, independentemente do tempo de profissão, há de se considerar as condições de trabalho em que os profissionais estão inseridos, pelo fato das más condições se constituir como agente facilitador ao desenvolvimento de estresse laboral.

Em relação às dimensões da Síndrome de Burnout, a Diminuição da Realização Pessoal foi a que apresentou maior escore médio, resultado que corrobora com o estudo de Mene- 
ghini; Paz e Lautert, (2011), o qual aponta a influência negativa da sobrecarga de trabalho na realização profissional.

Ao avaliar o contexto de trabalho da equipe de enfermagem da ESF encontrou-se como maior escore médio o fator Organização do Trabalho. Resultado este semelhante ao estudo de Santos e David (2011), o qual desenvolvido com enfermeiros de Unidade de Terapia Intensiva de um hospital privado do Rio de Janeiro.

A Organização do Trabalho é apontada como o fator que mais desmotiva a equipe de enfermagem, fica evidente que ocorre uma desintegração entre membros e processo de trabalho, no qual a prática e o ambiente de trabalho se tornam descaracterizados para o desenvolvimento de ações e práticas laborativas (David et al., 2009).

Os itens da avaliação do contexto de trabalho referentes à cobrança por resultados, ritmo de trabalho e pressão temporal para realização das tarefas foram os de maior escore médio. Este resultado demonstra que a cobrança por um desempenho quantitativo e qualitativo é elevada, devido à forte pressão existente nas instituições de saúde para a realização das tarefas, porém, há dificuldades reais no que tange às condições de trabalho para sua execução (Moisés; Medeiros; Freitas, 2013).Frequentemente, o ritmo acelerado e a cobrança por resultados são características de trabalho que sugerem riscos graves à saúde, potencializando o mal-estar no trabalho e o risco de adoecimento destes profissionais (Campos; David, 2011).

O fator Relações Socioprofissionais apresentou uma correlação forte com a dimensão Exaustão Emocional, o que evidencia que o convívio pautado no diálogo, na amizade, na coparticipação, dentro das equipes de saúde da família propiciam a concretização de um trabalho acolhedor (Azambuja et al., 2007). E que quando esse relacionamento não é satisfatório pode desencadear consequências emocionais negativas nos profissionais entrevistados.

O modo como o processo de trabalho é organizado pode interferir na realização do profissional para com o trabalho desempenhado, isso é demonstrado na correlação forte existente entre a Diminuição da Realização Pessoal e a Organização do Trabalho. Bertocini, Pires e Scherer (2011) afirmam que as inconformidades dos meios de trabalho inibem, ou até mesmo evitam, a realização da assistência de Saúde da Família, pautada na integralidade e promoção da saúde.

Organização do Trabalho também se correlacionou de modo moderado com a dimensão Despersonalização. Assunção e Jackson Filho (2011) questionam se o sentimento de queda da qualidade do serviço prestado está adoecendo os profissionais, ou se os profissionais adoecidos estão desestimulados para desempenharem seu serviço com qualidade nas unidades de saúde.

\section{CONCLUSÃO}

Na presente pesquisa que avaliou a influência do contexto de trabalho para o aparecimento da Síndrome de Burnout nos profissionais da equipe de enfermagem da ESF, os dados evidenciaram que quanto pior as condições de trabalho, organização do trabalho e as relações socioprofissionais maior a probabilidade de o profissional apresentar os sintomas característicos da Síndrome de Burnout.

Evidenciou-se que a dimensão Diminuição da Realização Pessoal foi a que apresentou maio escore médio, dentre as dimensões da Síndrome de Burnout. O fator Organização do Trabalho apareceu com maior escore médio no que concerne à avaliação do contexto de trabalho. 
Os autores compreendem que a presente pesquisa teve como limitação o fato da coleta ter sido realizada no ambiente de trabalho, uma vez que, por mais que a coleta tenha ocorrido em local privado, o ambiente de trabalho pode se constituir como fator interferente na resposta dos sujeitos.

Conhecer os fatores do contexto de trabalho que podem influenciar no aparecimento da Síndrome de Burnout é relevante para o planejamento de ações laborais, no intuito de minimizar os efeitos negativos advindos das mesmas. Ao promover um ambiente de trabalho com condições, organização e relações socioprofissionais adequadas reflete na qualidade da assistência oferecida ao usuário dos serviços de saúde.

\section{REFERÊNCIAS}

- Assunção, A. A. e Jackson Filho, J. M. (2011). Transformações do Trabalho no Setor Saúde e Condições para Cuidar. In A. A.Assunção e J. Brito (Eds.), Trabalhar na Saúde: experiências cotidianas e desafios para a gestão do trabalho e do emprego. (22), 45-66.

- Azambuja, E. et al. (2007). Significado do trabalho no processo de viver de trabalhadoras de um programa de saúde da família. Texto Contexto Enfermagem, 16(1), 7179.

- Bertocini, J. H., Pires, D. E. P. e Scherer, M. D. A. (2011). Condições de Trabalho e renormalizaçãoes nas atividades das enfermeiras na saúde da família. Trab. Educ. Saúde, 9(1), 157-173.

- Brasil. Portaria no 2.488, de 21 de outubro de 2011. (2011). Aprova a Política Nacional de Atenção Básica, estabelecendo a revisão de diretrizes e normas para a organização da Atenção Básica, para a Estratégia Saúde da Família (ESF) e o Programa de Agentes Comunitários de Saúde (PACS). Diário Oficial lda República Federativa do Brasil], Brasília.

- Campos, J. F. e David, H. S. L. (2011). Avaliação do contexto de trabalho em terapia intensiva sob o olhar da psicodinâmica do trabalho. Revista da Escola de Enfermagem da USP, 45(2), 363-368.

- Fernandes, M. C. P. e Backes, V. M. S. (2010). Educação em saúde: perspectivas de uma equipe da Estratégia Saúde da Família sob a óptica de Paulo Freire. Revista Brasileira de Enfermagem, 63(4), 567-73.

- Ferreira, A. C. e Mendes, A. M. (2008). Contexto de Trabalho. In M. M. M.Siqueira. (Ed.), Medidas do Comportamento Organizacional: Ferramentas de Diagnóstico e Gestão, 111-123.

- França, M. F. et al. (2012). Burnout e os aspectos laborais na equipe de enfermagem de dois hospitais de médio porte. Revista Latino Americana de Enfermagem, 20(5), 961-970.

- França, F. M. e Ferrari, R. (2012). Síndrome de Burnout e os aspectos sócio-demográficos em profissionais de enfermagem. Acta Paulista de Enfermagem, 25(5), 743-8.

- Holmes, E. S. et al. (2014). Burnout syndrome in nurses acting in primary care: an impact on quality of life. Cuidado é Fundamental Online, 6(4), 1384-1395. Disponível em: http://www.seer.unirio.br/index.php/cuidadofundamental/article/view/3311. Acesso em: 20 de Março de 2014 .

- Linch, G. F. C. e Guido, L. A. (2011). Estresse de enfermeiros em unidade de hemodinâmica no Rio Grande do Sul, Brasil. Revista Gaúcha de Enfermagem, 32(1), 63-71.

- Meneghini, F., Paz, A. A. e Lautert, L. (2011). Fatores ocupacionais associados aos componentes da síndrome de burnout em trabalhadores de enfermagem. Texto Contexto Enfermagem, 20(2), 225-233.

- Moisés, S. M., Medeiros, S. M. e Freitas, J. A. C. (2013). Influência do contexto de trabalho na saúde dos profissionais de enfermagem de uma unidade de terapia intensiva em um hospital universitário. Enferm. Glob,12(32).

- Santos, K. T. D. et al. (2011). Agente comunitário de saúde: perfil adequado a realidade do Programa Saúde da Família? Ciência \& Saúde Coletiva 16(1), 1023-1028.

- Santos, L. F. B. e David, H. M. S. L. (2011). Percepções do estresse no trabalho pelos agentes comunitários de saúde. Revista de enfermagem UERJ, 19(1), 52-57. 


\section{Cultura de las Cuidados}

- Silva-Roosli, A. C. B. D. y Athayde, M. (2011). Gestão, Trabalho e Psicodinâmica do reconhecimento no cotidiano da Estratégia em Saúde da Família In Trabalhar na Saúde: experiências cotidianas e desafios para gestão do trabalho e do emprego, (pp. 29-74).

- Tamayo, M.R. (1997). Relação entre a síndrome de bur- nout e os valores organizacionais no pessoal de enfermagem de dois hospitais públicos. Dissertação de Mestrado, Instituto de Psicologia, Universidade de Brasília.

- Trindade, L. L. e Lautert, L. (2010). Estresse e síndrome de burnout entre trabalhadores da equipe de Saúde da Família. Acta Paulista de Enfermagem. 23(5), 684-689.

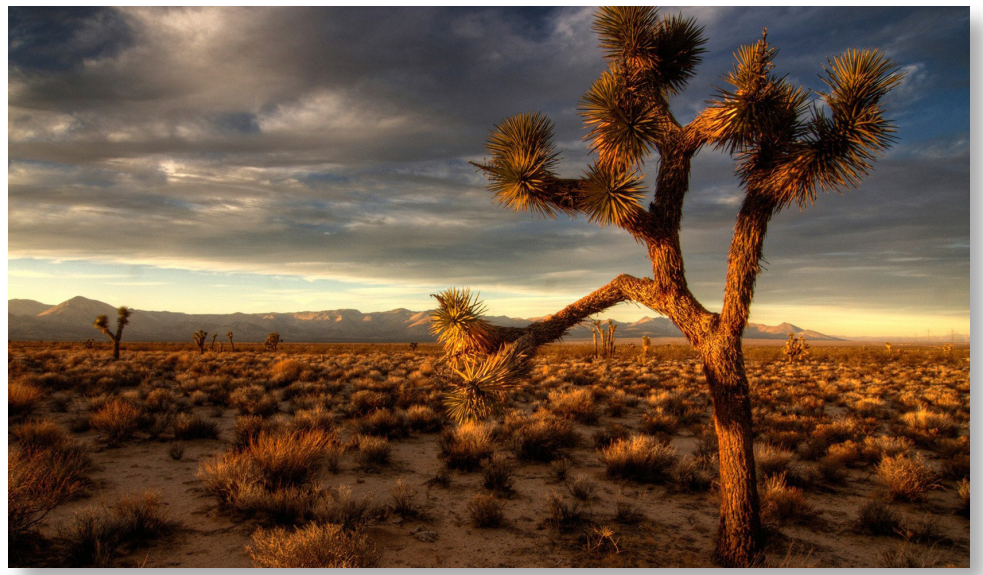

